\title{
High Dynamic Range and the Search for Planets
}

\author{
A. T. Tokunaga, C. Ftaclas, J. R. Kuhn, and P. Baudoz \\ Institute for Astronomy, Univ. of Hawaii, 2680 Woodlawn Dr., \\ Honolulu, HI 96822
}

\begin{abstract}
General arguments for optimized coronagraphy in the search for planets are presented. First, off-axis telescopes provide the best telescopic platforms for use with coronagraphy, and telescope fabrication technology now allows the fabrication of such telescopes with diameters of up to $6.5 \mathrm{~m}$. We show that in certain circumstances a smaller telescope with an off-axis primary has a signal-to-noise advantage compared with larger Cassegrain telescopes. Second, to fully exploit the advantages of the coronagraph for suppressing stray light, it is necessary to use a high Strehl ratio adaptive optics system. This can be best achieved initially with modest aperture telescopes of 3-4 $\mathrm{m}$ in diameter. Third, application of simultaneous differential imaging and simultaneous polarimetric techniques are required to reach the photon-limit of coronagraphic imaging. These three developments, if pursued together, will yield significant improvements in the search for planets.
\end{abstract}

\section{Introduction}

There has been much interest recently in the pursuit of planet detection using 8 and $10 \mathrm{~m}$ telescopes, primarily to take advantage of the higher angular resolution and greater light gathering power. The primary problem to overcome is the large amount of scattered light from the star in a region where planets might be found (within 1-2" radius of the star). Since the reflected light from a Jovian-size planet is about $10^{-9}$ that of the primary star's intensity, it is necessary to employ techniques to reduce the scattered light arising from Earth's atmosphere, secondary mirror spiders, the hole in the primary mirror, and from optical surfaces such as the primary and secondary mirrors of the telescope.

In this paper we review the advantages of off-axis telescopes and the advantages of searching for planets with a high-order adaptive optics (AO) systems on 3-4 m class telescopes. It is worth noting that some of the most significant astronomical discoveries in the past decade were made using modest aperture telescopes (about $2 \mathrm{~m}$ or smaller), including

- The discovery of the first brown dwarfs (Nakajima et al. 1995; Rebolo et al. 1995) and the subsequent discovery of field brown dwarfs by the 2MASS and DENIS surveys.

- The discovery of planetary mass objects (Mayor \& Queloz 1995) 
- The discovery of Kuiper Belt objects (Jewitt \& Luu 1993)

We suggest here an approach for the direct detection of planets by using modest aperture telescopes to develop techniques that can be scaled up to larger telescopes.

\section{Advantages of Off-Axis Telescopes}

A case for off-axis telescopes for research requiring high dynamic range has been made by Kuhn \& Hawley (1999). Here we summarize a few major points. Figure 1 shows the scattered light from a Cassegrain telescope. It illustrates the problems in attempting to detect planets near a bright star, namely saturation of the CCD (the vertical stripe), scattered light from the secondary spiders (cross feature at $45^{\circ}$ ), and scattered light from the atmosphere and mirrors.

Use of an occulting disk in the focal plane will eliminate the saturation effects of the point source. For the sources of scattered light Kuhn \& Hawley (1999) argue that an off-axis telescope will provide a greatly improved pointspread function (PSF). This is illustrated in Figure 2. The off-axis telescope provides an unobscured pupil image, thus eliminating scattered light components from the secondary spiders and the central hole in the primary mirror. In the search for planets, the relatively clean PSF of the off-axis telescope is a great practical advantage.

In attempting the direct imaging of planets, it is desirable to employ a coronagraph to suppress the light from the star. A comparison of the coronagraphic performance of a Cassegrain and off-axis telescope is shown in Figure 3. The calculation assumes scattering from the atmosphere and natural seeing (no AO). Although the gain is modest (about $0.5 \mathrm{mag}$ at the most), it should be noted that the scattered light from the secondary spiders is significant (see Figs. 1 and 2). We also show in Section 3 that further sensitivity gains can be made by using a high-order AO system.

There is a further advantage of the off-axis telescope with a coronagraph that arises from the significantly higher throughput in the off-axis telescope case. There are two items that affect the throughput. The first is that the encircled energy of the central core is less for a telescope with a central obscuration than one without a central obscuration. The second is that the throughput of a coronagraph for a cassegrain telescope is less than that of an off-axis telescope. These effects combine to make the integration time required to detect a faint nearby planet with an off-axis $4 \mathrm{~m}$ telescope competitive with that of an $8 \mathrm{~m}$ Cassegrain telescope for coronagraphy without adaptive optics. This is demonstrated below.

A Cassegrain telescope has an encircled energy in the diffraction-limited image core that decreases with the size of the linear obscuration of the secondary (Schroeder 1987). In Table 1 we show the encircled energy in the image core for an off-axis 4-m telescope, an off-axis 6-m telescope, and a Cassegrain 8-m 
telescope with a secondary having a linear obscuration of 0.25 (a $2 \mathrm{~m}$ diameter secondary). We show the encircled energy before the pupil mask of the coronagraph and after the pupil mask. A pupil mask that is 0.9 times the diameter of the primary mirror is assumed. Considering that the hole in the primary mirror must also be masked, the central obscuration increases from 0.25 to 0.40 in the case of the Cassegrain telescope, and the encircled energy is correspondingly reduced to 0.55 .

Table 1. Comparison of encircled energy

\begin{tabular}{c|c|c|c|c|c}
\hline \multicolumn{3}{c|}{ Before Lyot Mask } & \multicolumn{3}{c}{ After Lyot Mask } \\
\hline $\begin{array}{c}\text { Diameter } \\
(\mathrm{m})\end{array}$ & $\begin{array}{c}\text { Linear } \\
\text { Obscuration }\end{array}$ & $\begin{array}{c}\text { Encircled } \\
\text { Energy }\end{array}$ & $\begin{array}{c}\text { Diameter } \\
(\mathrm{m})\end{array}$ & $\begin{array}{c}\text { Linear } \\
\text { Obscuration }\end{array}$ & $\begin{array}{c}\text { Encircled } \\
\text { Energy }\end{array}$ \\
\hline 4 & 0.0 & 0.84 & 3.6 & 0.0 & 0.84 \\
6 & 0.0 & 0.84 & 5.4 & 0.0 & 0.84 \\
8 & 0.25 & 0.70 & 7.2 & 0.4 & 0.55 \\
\hline \hline
\end{tabular}

The reduction in encircled energy lowers the signal-to-noise in the detection of planets, that is, the peak flux in the planet PSF is reduced. Assuming background-limited imaging with $\mathrm{AO}$, the integration time is proportional to $\sim 1 /(A \phi)^{2}$, where $A$ is the effective collecting area and $\phi$ is the peak object intensity/background intensity. Table 2 shows the integration time ratios for a $4 \mathrm{~m}$ off-axis telescope to an $8 \mathrm{~m}$ Cassegrain telescope and for a $6 \mathrm{~m}$ off-axis telescope to an $8 \mathrm{~m}$ Cassegrain telescope. We see that after the coronagraph, the $4 \mathrm{~m}$ telescope requires 4 times longer integration, but this is much less time than the factor of 11 times longer before the coronagraph. The $6 \mathrm{~m}$ off-axis telescope is better than the $8 \mathrm{~m}$ cassegrain telescope after the coronagraph.

In the case of background-limited imaging and no $\mathrm{AO}$, the integration time is proportional to $\sim 1 /\left(A \phi^{2}\right)$, and the ratio of integration times is shown in the column 4 of Table 2 . In this case the $4 \mathrm{~m}$ off-axis telescope is competitive with an $8 \mathrm{~m}$ Cassegrain telescope (except for the smaller diffraction-limited core of the 8 $m$ telescope). These calculations also do not consider the effect of the scattered light from the secondary spiders, as mentioned above. These arguments were previously made by Ftaclas (1994). We therefore conclude that using off-axis telescopes for the detection of planets should be seriously considered.

Table 2. Comparison of integration time

\begin{tabular}{c|c|c|c}
\hline $\begin{array}{c}\text { Integration } \\
\text { Time Ratio }\end{array}$ & $\begin{array}{c}\text { Before } \\
\text { Coronagraph }\end{array}$ & $\begin{array}{c}\text { After } \\
\text { Coronagraph }\end{array}$ & $\begin{array}{c}\text { No Adaptive } \\
\text { Optics }\end{array}$ \\
\hline $\mathrm{t}(4 \mathrm{~m}) / \mathrm{t}(8 \mathrm{~m})$ & 11 & 4 & 1.3 \\
$\mathrm{t}(6 \mathrm{~m}) / \mathrm{t}(8 \mathrm{~m})$ & 2.2 & 0.74 & 0.75 \\
\hline \hline
\end{tabular}

\section{The Case for High-Order AO on "Small" Telescopes}

Lai (2001) and Oppenheimer, Sivaramakrishnan, \& Makidon (2002) make the case for pursuing high-order AO on 3-4 m class telescopes. We follow the argu- 
ments of Oppenheimer et al. here, since they address specifically the question of detecting planets. For a given number of actuators on the AO system, higher spatial frequencies will be corrected by the AO system on a smaller telescope than on a larger telescope. Therefore the Strehl ratio (SR) will be higher on the smaller telescope. The contrast improvement is

$$
\eta=\frac{S_{\mathrm{AO}}}{S_{\mathrm{see}}}\left(1-S_{\mathrm{AO}}\right)^{-1}
$$

where $\mathrm{S}_{\mathrm{AO}}$ is the $\mathrm{SR}$ of the AO system and $\mathrm{S}_{\text {see }}$ is the $\mathrm{SR}$ of the seeing limited image. The first term is the increase in the contrast of the image, and the second term is the increase in the energy from the wings to the core. It is evident that the contrast improvement is an extremely sensitive function of the SR of the AO system. Oppenheimer et al. argue that a very high SR on a 3-4 m telescope opens up an observational niche between the direct imaging of planets without adaptive optics and the indirect imaging of planets using Doppler-shift methods.

What SR is required to achieve this observational niche? Oppenheimer et al. find that for a $3.6 \mathrm{~m}$ telescope, a SR of $95 \%$ is a desirable goal. To reach this high SR, approximately 34 actuators across the aperture of the telescope (assuming a Shack-Hartmann wavefront sensor) are required. More actuators would begin to decrease the SR because there would be less photons for each element of the wavefront sensor. Furthermore, other effects such as atmospheric scintillation and speckle noise can become serious limitations at very high SRs. The former has not been addressed in a practical system as yet, whereas the latter is presently being addressed in several astronomical systems, as discussed in Section 4.

There is another important advantage of a high-order AO system. As the number of actuators increases, the AO system will be able to correct higher frequency wavefront errors. The spatial frequency cutoff is given by $k_{\mathrm{AO}}=$ $N_{\text {act }} / 2 D$, where $N_{\text {act }}$ is the number of actuators across the diameter of the primary mirror and $D$ is the diameter of the telescope. In the image plane this corresponds to an angle on the sky, $\theta_{\mathrm{AO}}=N_{\mathrm{act}} \lambda / 2 D$, over which the AO system is able to provide wavefront correction. This is illustrated in Figure 4. The most critical region for searching for planets around nearby stars would be at angular distances less than $2^{\prime \prime}$ from the star, a region that is mostly covered with $N_{\text {act }}=34$ on a $3.6 \mathrm{~m}$ telescope.

Note that Lai (2001) presents simulations that show curvature sensing AO systems can achieve a SR $>90 \%$ at $\mathrm{K}$ with as little as 104 actuators on a 3.6 $\mathrm{m}$ telescope. This suggests that further development of curvature systems is warranted.

Since a coronagraph typically has an occulting disk that is around $4 \lambda / D$ in diameter or larger, the region that is accessible with AO wavefront correction can be very small if $N_{\text {act }}$ is small or if the telescope aperture is large. Present coronagraphic systems have not met expectations through a combination of $N_{\text {act }}$ being too small or not having a way to reduce speckle noise. We can address the former problem by building a high-order $\mathrm{AO}$ system on a modest aperture telescope, but we must still deal with the speckle noise problem which is discussed in Section 4. 


\section{Further Scattered Light Suppression}

One must achieve photon noise limited imaging in the search for planets. In practice, $\mathrm{AO}$ observations are limited by speckle noise, the noise resulting from the residual uncorrected wavefront errors. Techniques have therefore been pursued to employ differential imaging to reduce the speckle pattern by subtracting images obtained simultaneously at two wavelengths. This technique relies on the search for spectral features that are different at the two wavelengths so that a residual signal from the planet can be detected (see Ftaclas 2003). A further refinement of this technique using three wavelengths is discussed by Marois, Doyon, \& Nadeau (2003). Practical problems include small differences in the speckle pattern resulting from differences in the wavelengths, so it is still not possible to reach the photon noise limit.

Another approach is to seek a polarization signature by observing all of the Stokes parameters simultaneously, as demonstrated by Kuhn, Potter, \& Parise (2001a). They show the feasibility of this approach. More extensive observations using a 36-element AO system on the Gemini North Telescope has shown that one can approach the photon-noise limit (Potter, private comm.).

One or both of these techniques employed with a coronagraph will have to be employed to detect planets. It is critical to reach the photon-noise limit, and further work to demonstrate that this can be achieved is required.

\section{A Development Path}

What is required to take the next step is to combine these ideas into a practical system. There is already a $\mathrm{N}_{\text {act }}=34$ system on the $3.7 \mathrm{~m}$ AEOS telescope on Haleakala. This could be exploited if the performance of the system is as good as predicted and a coronagraph is built to exploit it (Oppenheimer et al. 2003). Alternatively a 3-4 m class off-axis telescope with a high order AO system could be built to pursue the ideas presented here. The practical limitations of speckle noise and atmospheric scintillation would have to be overcome, but such a facility could demonstrate techniques to achieve photon noise limited imaging with a coronagraph and take advantage of the observational niche for searching for planets as described by Oppenheimer et al. (2002).

It is clear that going to larger off-axis telescopes would have the advantages of the larger collecting area and smaller diffraction-limited core. The calculations shown in Table 2 show that a $6 \mathrm{~m}$ off-axis telescope would be superior to a Cassegrain $8 \mathrm{~m}$ telescope on integration time considerations alone. It is also clear that an off-axis telescope would have the advantage of a cleaner PSF, as demonstrated in Figure 2. Therefore the next logical step would be to go to the $6 \mathrm{~m}$ size to fully exploit the advantages described here with a single aperture telescope. Such a facility would be the premier ground-based infrared telescope because an unobscured aperture would also provide minimum telescope emissivity.

In the future, it may be possible to build a multiple aperture large telescope using off-axis mirrors which would have advantages over large segmented mirror telescopes. This is discussed in detail by Kuhn et al. (2001b). 


\section{Summary}

We have summarized ideas that could significantly advance the detection of planets around nearby stars. Realization of these ideas would allow observations near bright stars that are limited by photon noise rather than scattered light and speckle noise. There are two key points:

1. Unobscured apertures (off-axis telescopes) provide higher throughput and a cleaner PSF.

2. High-order AO systems greatly enhance the performance of coronagraphs. Implementation of high-order AO systems on modest aperture telescopes (3-4 m) will initially provide an observational niche for detecting planets. Techniques to suppress speckle noise will be required to approach the photon noise limit.

\section{References}

Ftaclas, C. 1994, in Infrared Tools for Solar Astrophysics, What's Next?, ed. J. R. Kuhn \& M. J. Penn (Singapore: World Scientific)

Ftaclas, C., Martín, E. L., \& Toomey, D. 2003, this volume

Jewitt, D. C., \& Luu, J. X. 1993, Nature, 362, 730

Kuhn, J. R., \& Hawley, S. L. 1999, PASP, 111, 601

Kuhn, J. R., Potter, D., \& Parise, B. 2001a, ApJ, 553, L189

Kuhn, J. R., Moretto, G., Racine, R., Roddier, F., \& Coulter, R. 2001b, PASP, 113,1486

Lai, O. 2001, in Beyond Conventional Adaptive Optics, ed. R. Ragazzoni, N. Hubin, \& S. Esposito (Venice: ESO)

Marois, C., Doyon, R., \& Nadeau, D. 2003, this volume

Mayor, M., \& Queloz, D. 1995, Nature, 378, 355

Nakajima, T., Oppenheimer, B. R., Kulkarni, S. R., Golimowski, D. A., Matthews, K., \& Durrance, S. T. 1995, Nature, 378, 463

Oppenheimer, B. R., Sivaramakrishnan, \& Makidon, R. B. 2002, in The Future of Small Telescopes, ed. T. Oswalt (Dordrecht: Kluwer)

Oppenheimer, B. R., et al. 2003, this volume

Rebolo, R., Zapatero-Osorio, M. R., \& Martín, E. L. 1995, Nature, 377, 129

Schroeder, D. 1987, Astronomical Optics (San Diego: Academic Press), 185

Sivaramakrishnan, A., Koresko, C. D., Makidon, R. B., Berkefeld, T., \& Kuchner, M. J. 2001, ApJ, 552, 397 


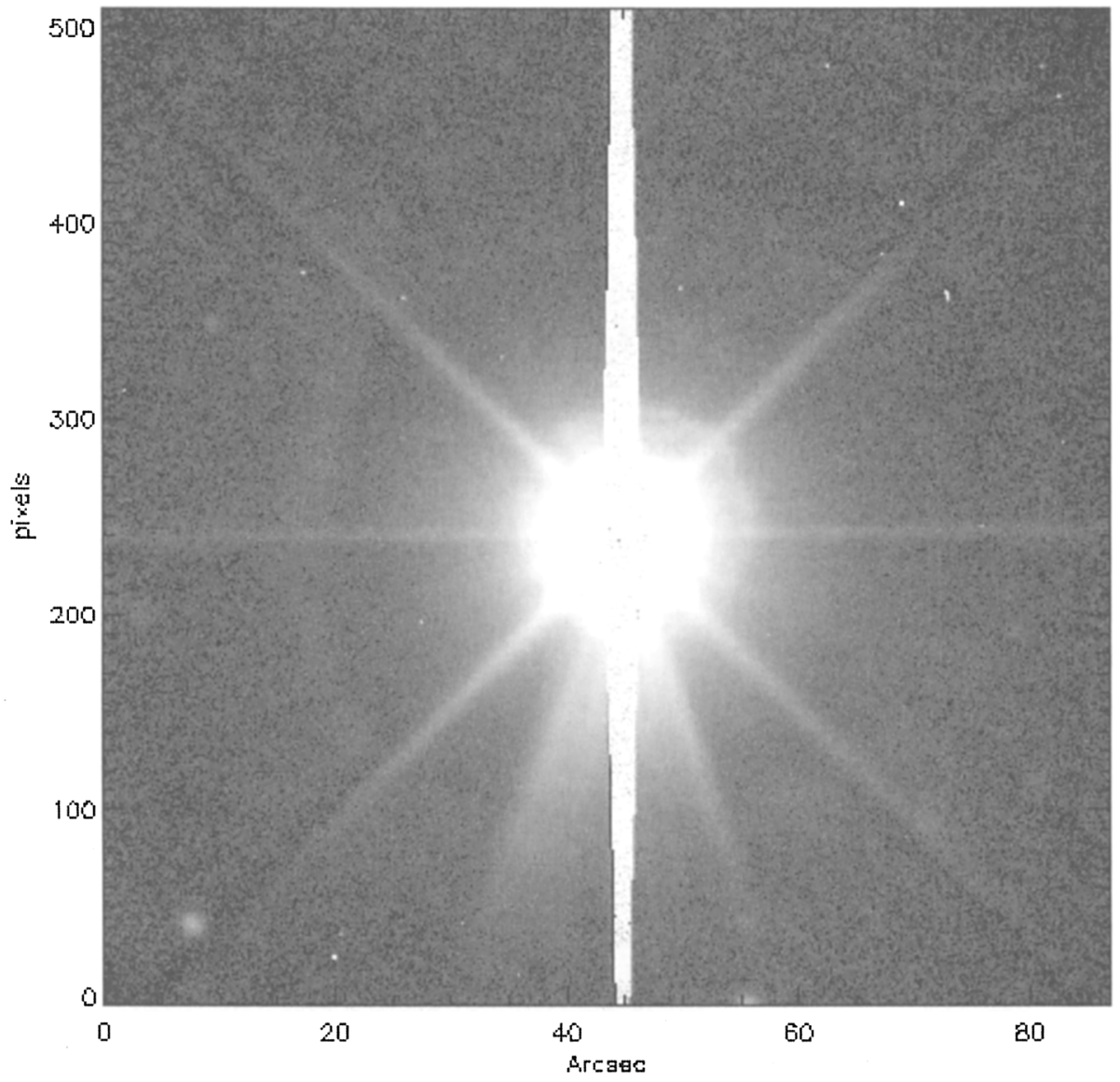

Figure 1. Scattered light from the Blanco telescope. Adapted from Kuhn \& Hawley (1999). 

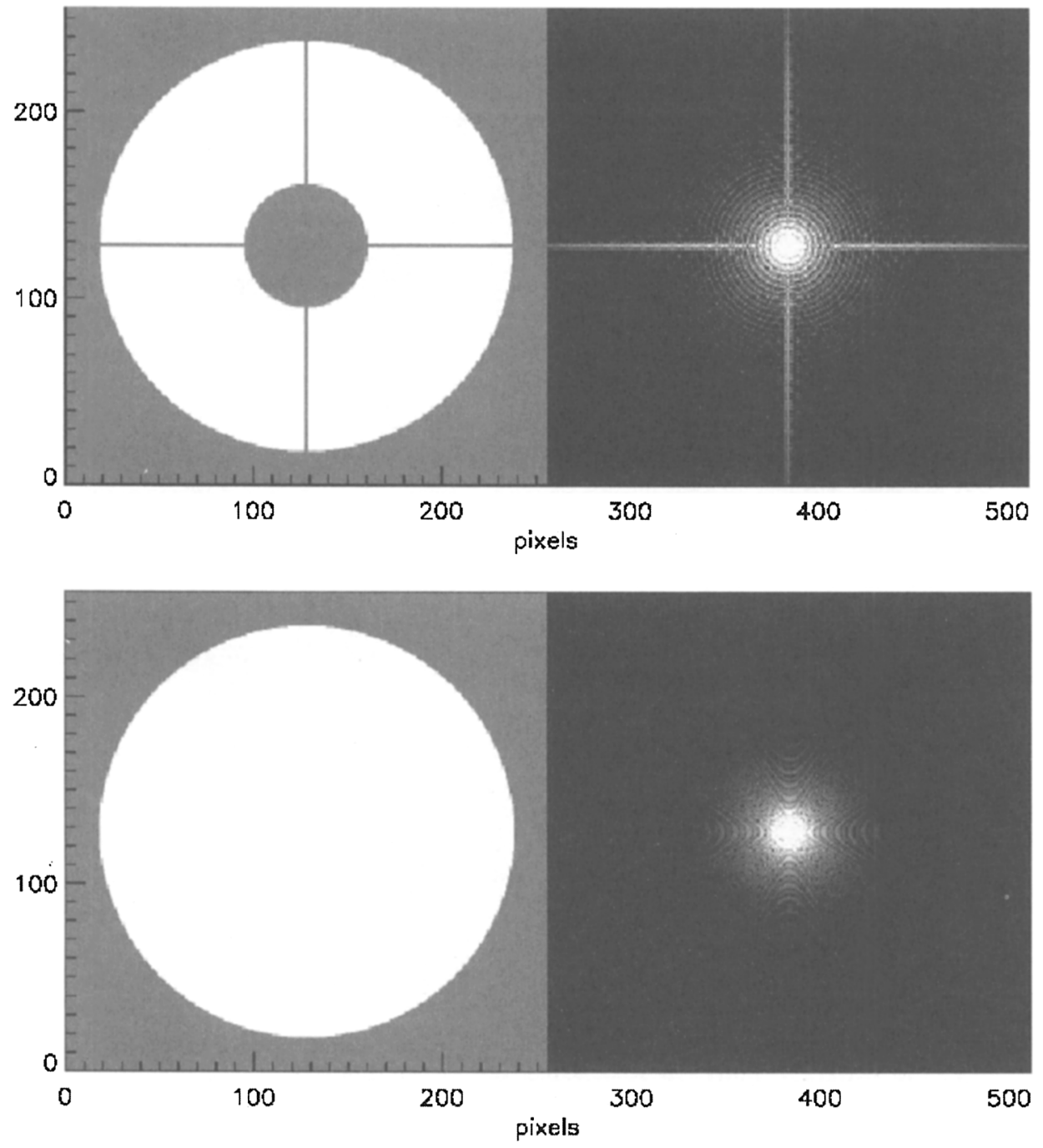

Figure 2. Comparison of obscured and unobscured pupil images. Adapted from Kuhn \& Hawley (1999). 


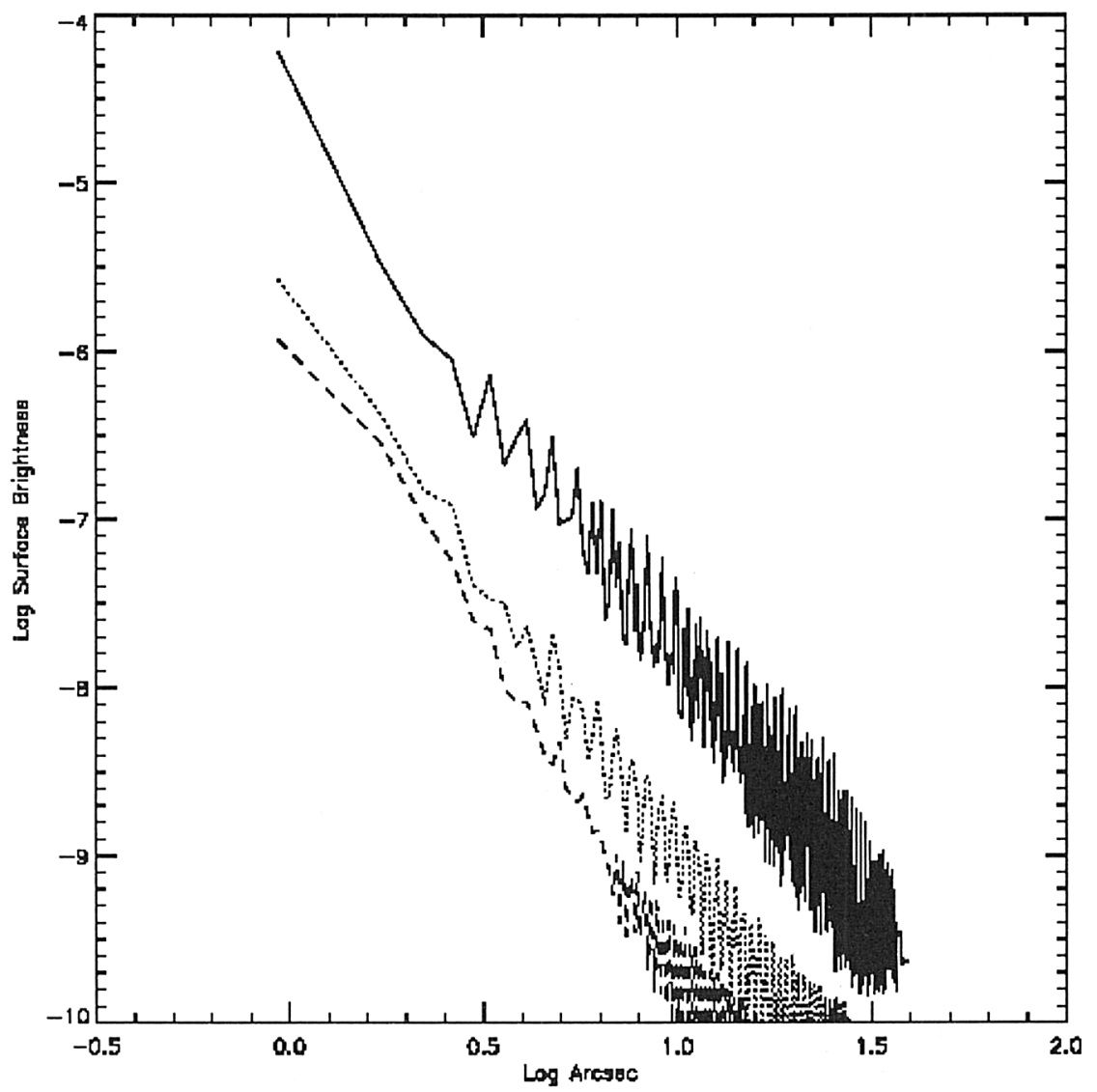

Figure 3. Comparison of coronagraphic performance of a Cassegrain telescope to that of an off-axis telescope. The upper curve shows the radial surface brightness profile of the direct image, the middle curve shows that of a Cassegrain coronagraphic PSF, and the lower curve shows that of an off-axis telescope coronagraphic PSF. Adapted from Fig. 14 of Kuhn \& Hawley (1999). 


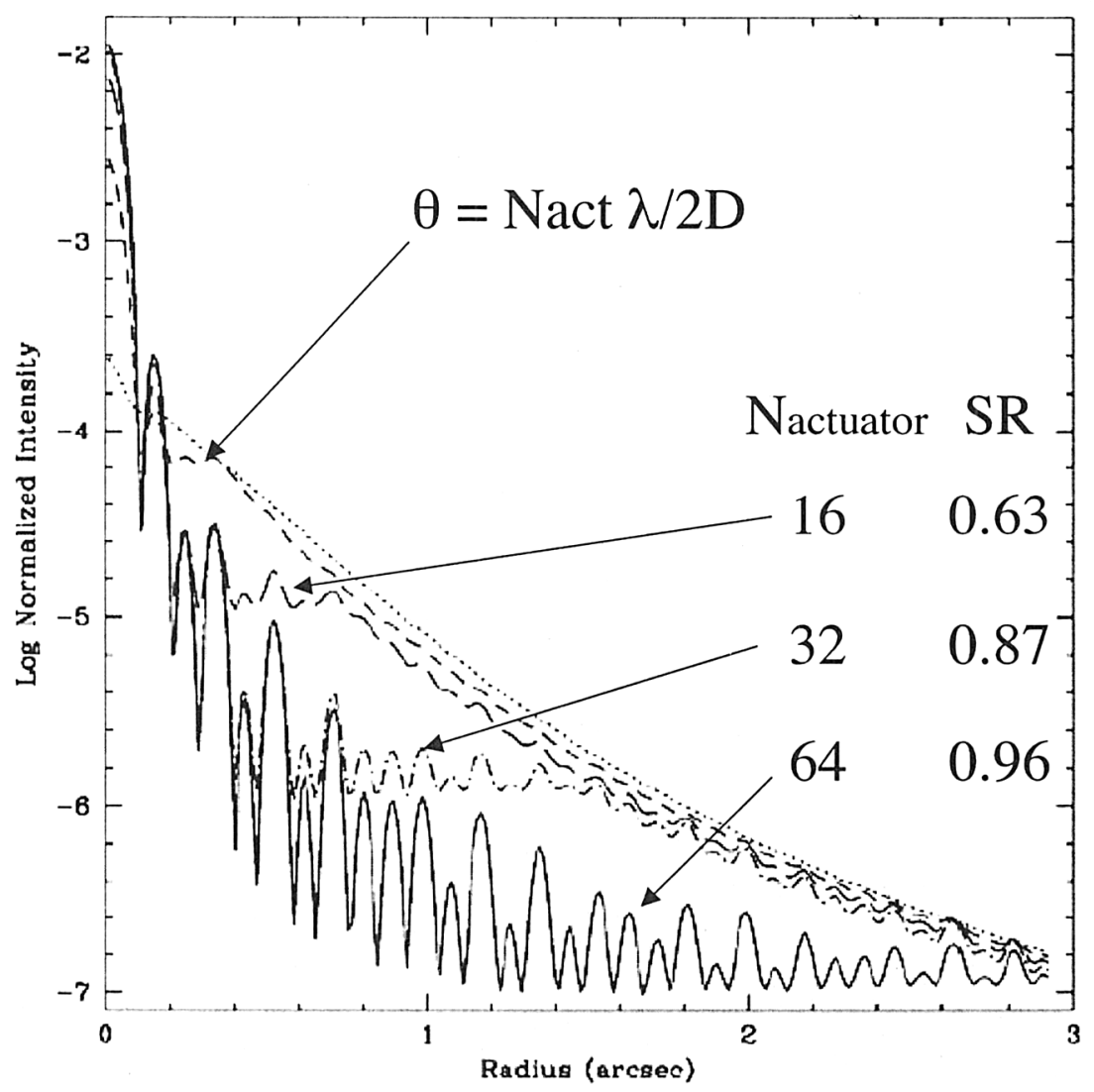

Figure 4. Radial profiles of simulated AO PSF (assuming a ShackHartmann wavefront sensor). $N_{\text {act }}$ is the number of actuators across the primary mirror. Adapted from Oppenheimer et al. (2002). See also Sivaramakrishnan et al. (2001). 